\title{
Neoadjuvant intraperitoneal and systemic paclitaxel combined with apatinib and S-1 chemotherapy for conversion therapy in gastric cancer patients with positive exfoliative cytology: a prospective study
}

\author{
Ping'an Ding, Peigang Yang, Yuan Tian, Honghai Guo, Yang Liu, Ze Zhang, Tao Zheng, Bibo Tan, \\ Zhidong Zhang, Dong Wang, Yong Li, Qun Zhao
}

The Third Department of Surgery, the Fourth Hospital of Hebei Medical University, Shijiazhuang, China

Contributions: (I) Conception and design: Q Zhao; (II) Administrative support: Q Zhao; (III) Provision of study materials or patients: P Ding, P Yang, Y Tian, H Guo, Y Liu, Z Zhang, T Zheng, B Tan, Z Zhang, D Wang, Y Li; (IV) Collection and assembly of data: P Ding, P Yang, Y Tian, H Guo; (V) Data analysis and interpretation: P Ding; (VI) Manuscript writing: All authors; (VII) Final approval of manuscript: All authors.

Correspondence to: Qun Zhao. The Third Department of Surgery, the Fourth Hospital of Hebei Medical University, Shijiazhuang 050011, China. Email: zhaoqun@hebmu.edu.cn.

Background: To explore the efficacy and safety of neoadjuvant intraperitoneal and systemic (NIPS) paclitaxel chemotherapy combined with apatinib and S-1 in the treatment of gastric cancer patients with positive exfoliative cytology.

Methods: Patients with gastric cancer $\left(\mathrm{P}_{0} \mathrm{CY}_{1}\right)$ who were confirmed to have free cancer cells (FCCs) in the abdominal cavity after laparoscopic exploration from April 2018 to August 2019 were enrolled. All patients underwent NIPS chemotherapy using paclitaxel combined with apatinib and S-1 treatment. Laparoscopic exploration was performed after 3 cycles of conversion therapy. The primary study endpoint was the FCC negative rate, and the secondary study endpoints were overall survival time (OS), progression-free survival time (PFS), objective response rate (ORR), disease control rate (DCR), and safety indicators.

Results: Out of 312 advanced gastric cancer patients who underwent laparoscopic exploration, 36 patients with $\mathrm{P}_{0} \mathrm{CY}_{1}$ gastric cancer were identified and enrolled in this study. After 3 cycles of conversion therapy, the ORR was $80.56 \%$ and the DCR was $94.44 \%$. All patients underwent secondary laparoscopic exploration, and the FCC conversion rate was $77.78 \%$. All patients with negative FCC underwent R0 surgical resection, with a median follow-up time of 11.4 months. The median survival time was 15.5 months, and the 1 -year OS was $80.55 \%$. The median PFS was 14.4 months, and the 1 -year PFS was $75.00 \%$. Treatment-related grade 3 adverse reactions were mainly leukopenia and neutropenia. No grade 4 adverse reactions were observed. There were no reported deaths related to chemotherapy or surgery in the study cohort.

Conclusions: NIPS with paclitaxel combined with apatinib and S-1 treatment may increase the FCC negative rate of $\mathrm{P}_{0} \mathrm{CY}_{1}$ gastric cancer patients.

Keywords: Apatinib; abdominal exfoliation cytology positive; gastric cancer; neoadjuvant intraperitoneal and systemic treatment (NIPS treatment)

Submitted Jun 08, 2021. Accepted for publication Jul 29, 2021.

doi: 10.21037/jgo-21-375

View this article at: https://dx.doi.org/10.21037/jgo-21-375 


\section{Introduction}

In 2020 , there are about 769,000 deaths due to gastric cancer worldwide, ranking fourth in the number of deaths from malignant tumors, of which $43.9 \%$ and $48.6 \%$ of deaths occur in China (1). Gastric cancer causes significant morbidity and mortality in China. At the time of diagnosis, most patients are already in the advanced stages of the disease. Despite effective and radical removal of local lesions, patient prognosis remains poor (2). This is largely due to the presence of undetected metastases and free cancer cells (FCCs) in the abdominal cavity before surgery, which leads to implantation and metastasis after surgery.

Therefore, the key to success in advanced gastric cancer surgery is to remove any potentially hidden metastases in the abdominal cavity (3). Although systemic intravenous chemotherapy regimens have been shown to improve the prognosis of patients in some cases, the presence of the peritoneal-plasma barrier makes it difficult for chemotherapeutics with larger molecular sizes to penetrate the barrier and to act on peritoneal micrometastases or metastases within the peritoneal cavity. Therefore, systemic chemotherapy has little effect on peritoneal metastasis (4). The positive exfoliative cytology gastric cancer $\left(\mathrm{P}_{0} \mathrm{CY}_{1}\right)$ is a special type of advanced gastric cancer, and previous studies have not analyzed such patients alone. Meanwhile, there is still lack of internationally recognized standard regimens for the treatment of $\mathrm{P}_{0} \mathrm{CY}_{1}$ gastric cancer patients.

In recent years, studies have reported that the combined application of intraperitoneal and systemic chemotherapy achieved good results in gastric cancer peritoneal metastases (5-8). However, there have been few studies examining conversion therapy in patients with cytologypositive peritoneal lavage fluid $\left(\mathrm{CY}_{1}\right)$ without macroscopic peritoneal metastasis $\left(\mathrm{P}_{0}\right)\left(\mathrm{P}_{0} \mathrm{CY}_{1}\right)$ gastric cancer. Furthermore, there is a paucity of data on the combined application of targeted drugs to treat such patients. Apatinib is a novel, small molecule anti-angiogenesis inhibitor that can be administered orally. A series of clinical studies (9-12) demonstrated that apatinib exerted a certain objective effectiveness and obvious survival benefit in patients with advanced gastric cancer, with a low incidence of serious adverse effects and good tolerance. Therefore, this current study was conducted to explore the short-term clinical efficacy and safety of NIPS paclitaxel chemotherapy combined with apatinib and the oral fluoropyrimidine derivative $\mathrm{S}-1$ in the treatment of patients with $\mathrm{P}_{0} \mathrm{CY}_{1}$ gastric cancer. We present the following article in accordance with the TREND reporting checklist (available at https://dx.doi.org/10.21037/jgo-21-375).

\section{Methods}

\section{Clinical data}

A total of 36 patients with $\mathrm{P}_{0} \mathrm{CY}_{1}$ gastric cancer were enrolled in this single-center, prospective, single-arm phase II clinical trial (NCT03718624). The following inclusion criteria were applied: (I) gastric adenocarcinoma confirmed by histopathology and FCC positivity confirmed by exfoliated cells in the abdominal cavity; (II) preoperative computed tomography (CT) imaging showed no distant organ metastasis and no distant lymph node metastasis above the third station; (III) patients aged $\leq 75$ years; (IV) the Eastern Cooperative Oncology Group (ECOG) activity status score was $\leq 2$ points; (V) patients had good bone marrow function, liver function, heart function, and kidney function, and were able to tolerate chemotherapy; (VI) there were no other serious immunosuppressive diseases or simultaneous malignant tumors; (VII) and pathological human epidermal growth factor receptor 2 (HER2) tests were negative prior to the operation. Patients were excluded if they presented with the following: (I) difficulty taking oral medications (such as inability to swallow, chronic diarrhea, and gastrointestinal obstruction, etc.); (II) high blood pressure that could not be controlled by a single antihypertensive drug treatment; (III) 24 hour urine protein quantification $>1.0 \mathrm{~g}$; (IV) imaging results showing the tumor had invaded important blood vessels or the investigator judged that the tumor was highly likely to invade important blood vessels during treatment and cause fatal bleeding; (V) abnormal blood coagulation; and (VI) other comorbidities that may seriously endanger the safety of the patient or affect the completion of the study as determined by the investigator. The study was conducted in accordance with the Declaration of Helsinki (as revised in 2013). This study was approved by the Ethics Committee of the Fourth Hospital of Hebei Medical University (Ethics number: 2018088), and all patients or their families provided informed consent.

\section{Study methods}

\section{Laparoscopic exploration}

The patient was placed in a supine position. An observation hole was established under the umbilicus with an open 

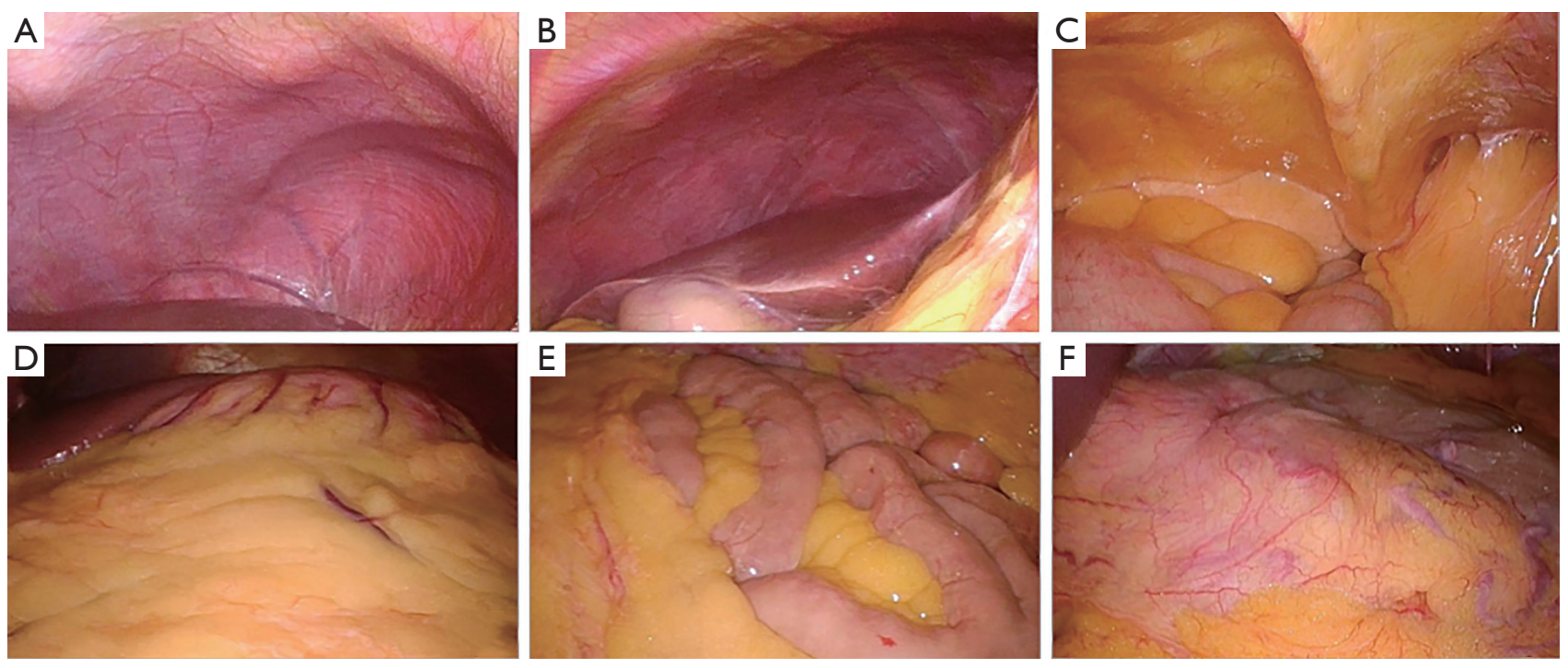

Figure 1 Laparoscopic exploration. (A) Diaphragm; (B) liver; (C) pelvic cavity; (D) omentum; (E) small mesenteric; (F) cancer.

method, and a $5 \mathrm{~mm}$ Trocar was placed on the left and right clavicular midline of the umbilical bone under direct vision. Operating forceps were placed for exploration and lavage. The sequence of abdominal cavity exploration was as follows: left and right under the diaphragm $\rightarrow$ liver, spleen $\rightarrow$ abdominal parietal peritoneum $\rightarrow$ pelvic cavity $\rightarrow$ greater omentum, small intestine and mesentery $\rightarrow$ transverse colon mesentery $\rightarrow$ stomach. The following were assessed during exploration: the presence of ascites; the presence of metastases in the abdominal and pelvic peritoneum, mesenteric, omentum and Douglas cavity; the presence of metastasis on the liver surface; swelling of the perigastric lymph nodes; infiltration of the gastric serosal surface; and rigidity of the stomach. The abdominal cavity was washed and exfoliation cytology examination was performed. Sterile normal saline $(500 \mathrm{~mL})$ was used to wash the left and right sides under the diaphragm, the abdominal pelvic peritoneum, and the mesentery. The peritoneal lavage fluid was collected from the Douglas cavity, under the liver, and from the spleen fossa. Samples were centrifuged and cytology was performed to detect the presence of any free tumor cells. For tumors on the posterior wall of the gastric body, the gastric colon ligament was cut and the small omental sac was explored, including the transverse mesocolon and the pancreatic capsule (Figure 1).

\section{Assessment of the peritoneal exfoliated cytology (13)}

Two professional physicians in the pathology department assessed the cytology results from the abdominal exfoliated cells. Positive FCCs were indicated by large nuclear staining with a nucleoplasmic ratio imbalance; nuclear chromatin that was dense, rough, and unevenly distributed, with disordered arrangement; thickened nuclear membrane that was interrupted or wrinkled; and increased nucleoli (Figure 2).

\section{Indwelling surgery in the abdominal wall chemotherapy port}

The chemotherapy port is a special port for the abdominal cavity with a larger caliber, including a base, a connecting loop, a puncture device, and a chemotherapy tube (15F$420 \mathrm{~mm}$ ). The port seat is usually placed at the middle and outer $1 / 3$ of the line connecting the anterior superior iliac spine and the umbilicus to ensure that the bottom of the base can play a supporting role. For the surgical incision (take 3-4 cm for unobstructed length), an oblique incision is selected on the outside of the port seat placement area, the skin and subcutaneous fat are cut, the fat layer and the fascia layer are separated, bleeding is stopped, and the chemotherapy port is placed $1.0-1.5 \mathrm{~cm}$ away from the epidermis. In addition, the surrounding tissues are fully freed inside the incision to form a port cavity, so that the abdominal wall chemotherapy port can be placed completely. The puncture rod is then inserted in the direction of the pelvic cavity to form a tunnel, and the abdominal wall chemotherapy tube is inserted (usually 12$15 \mathrm{~cm}$ ) along the tunnel, and connected to the port holder 


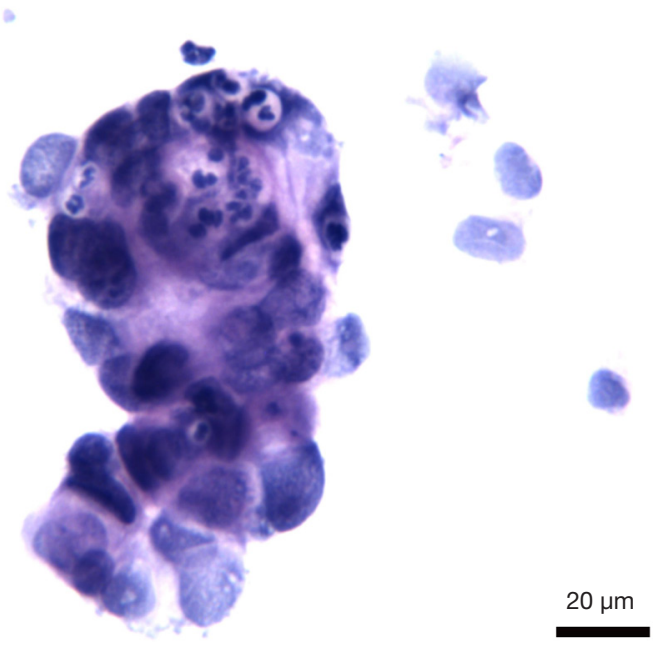

Figure 2 Microscopic examination of adenocarcinoma cells in the peritoneal irrigation fluid (HE staining, 400× magnification).

and the chemotherapy tube through a connecting loop, the head of the chemotherapy tube is clamped into the pelvic cavity with the aid of a laparoscope, and non-absorbable silk thread is used to fix the surrounding area of the port holder with the ribs while ensuring that the catheter is not bent. The membrane layer, the subcutaneous fat, and the skin are sequentially closed, and the patency of the port seat and catheter can then be tested.

\section{Chemotherapy regimen}

Treatment commenced on the day after the laparoscopic exploration, and each course of treatment lasted for 3 weeks. On the 1st and 8th day of the treatment course, paclitaxel was infused via an intraperitoneal (IP) chemotherapy pump (IP route $20 \mathrm{mg} / \mathrm{m}^{2}$, dissolved in $1,000 \mathrm{~mL}$ of normal saline, infusion for more than 1 hour) and intravenously (IV) (IV route $50 \mathrm{mg} / \mathrm{m}^{2}$, dissolved in $500 \mathrm{~mL}$ of saline, infusion for more than 1 hour). Dexamethasone and cimetidine were administered before paclitaxel treatment. Oral S-1 $80 \mathrm{mg} /\left(\mathrm{m}^{2} \cdot \mathrm{d}\right)$ was given 30 minutes after breakfast and 30 minutes after dinner for 14 consecutive days. At the same time, apatinib $500 \mathrm{mg} / \mathrm{d}$ was administered orally for 21 consecutive days. The dose of S-1 was determined according to the body surface area (BSA) as follows: for BSA $<1.25 \mathrm{~m}^{2}, 80 \mathrm{mg} /\left(\mathrm{m}^{2} \cdot \mathrm{d}\right) \mathrm{S}-1$ was administered; for BSA $1.25-1.50 \mathrm{~m}^{2}, 100 \mathrm{mg} /\left(\mathrm{m}^{2} \cdot \mathrm{d}\right) \mathrm{S}-1$ was administered; and for $\mathrm{BSA}>1.50 \mathrm{~m}^{2}, 120 \mathrm{mg} /\left(\mathrm{m}^{2} \cdot \mathrm{d}\right) \mathrm{S}-1$ was given (Figure 3).
The use of abdominal wall chemotherapy port

To use the abdominal wall chemotherapy port, the following procedures were followed: (I) after disinfecting the skin $10 \mathrm{~cm}$ from the inside to the outside with the harbor seat as the center, press down firmly with the thumb and index finger of the left hand until the contour of the harbor seat does not move but still has enough stable support to ensure the precise direction of the needle; (II) insert the butterfly wing liquid needle vertically into the center of the chemotherapy port area until it is difficult to insert the needle and the needle tip meets a hard barrier (the chassis of the port seat), fix the butterfly wing needle using a sterile transparent applicator; (III) give $100 \mathrm{~mL}$ normal saline instillation, confirm that the infusion has no resistance, confirm the patency of the pipeline, and observe whether there is any fluid leakage at the tip of the needle; (IV) after confirming that the fluid can enter the abdominal cavity smoothly, administer the paclitaxel injection; (V) after all treatments, instill $100 \mathrm{~mL}$ saline, remove the needle vertically, disinfect, and apply the dressing.

\section{Evaluation criteria}

Assessment of adverse reactions due to chemotherapy The common terminology criteria for adverse events (CTCAE) version 3.0 was adopted to assess the classification of adverse reactions (14).

\section{Evaluation criteria for chemotherapy remission}

The Response Evaluation Criteria in Solid Tumors (RECIST) version 1.1 was used to evaluate the efficacy of chemotherapy (15). Complete response (CR) is the complete disappearance of the target lesion after chemotherapy. Partial response (PR) is a reduction in the total length of all target lesions by $30 \%$ or more. Progressive disease (PD) is where the sum of the long diameters of all target lesions increase by at least $20 \%$ and the absolute value of the increase is more than $5 \mathrm{~mm}$, or the appearance of other new lesions is detected. Stable disease (SD) describes cases where the target lesion changes are unchanged. CR and PR cases are defined as effective chemotherapy, while SD and PD patients are defined as chemotherapy ineffective cases.

\section{Evaluation criteria for pathological remission}

The classification is based on the tumor regression grade (TRG) classification of the American Joint Committee on 


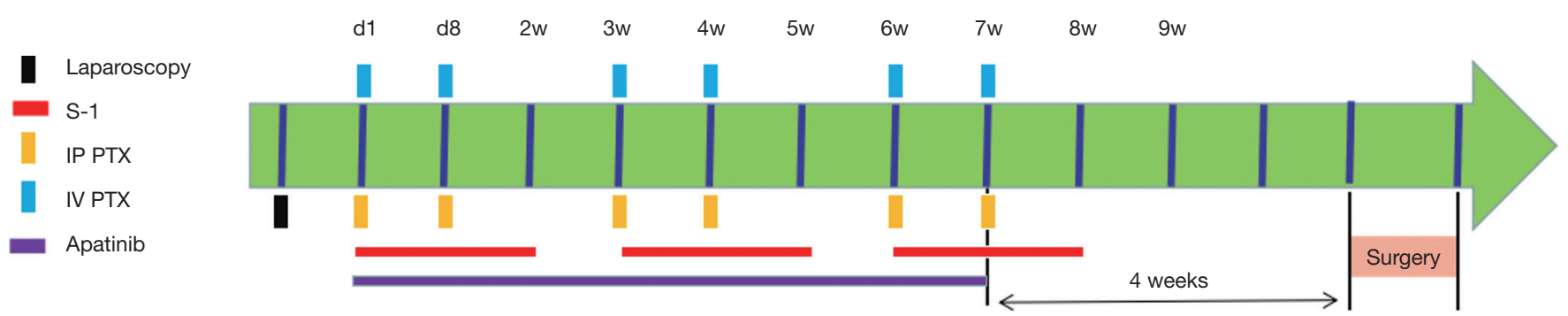

Figure 3 The specific medication regimen of 1 cycle of chemotherapy. On the 1st and 8th day of the treatment course, paclitaxel was infused via an intraperitoneal chemotherapy pump (IP route $20 \mathrm{mg} / \mathrm{m}^{2}$, dissolved in 1,000 $\mathrm{mL}$ normal saline, infusion for more than 1 hour), and intravenously (IV route $50 \mathrm{mg} / \mathrm{m}^{2}$, dissolved in $500 \mathrm{~mL}$ saline, infusion for more than 1 hour). Dexamethasone and cimetidine were administered prior to paclitaxel treatment. Orally S-1 $80 \mathrm{mg} /\left(\mathrm{m}^{2} . \mathrm{d}\right)$ was administered 30 minutes after breakfast and 30 minutes after dinner for 14 consecutive days. Drug administration was stopped 7 days. At the same time, apatinib (500 mg/d) was administered orally for 21 consecutive days. d, day; w, week; PTX, paclitaxel; IP, intraperitoneal; IV, intravenous.

Cancer Staging (AJCC) as follows: TRG level 0 represents complete retraction, no tumor cells visible under the microscope; TRG level 1 represents moderate regression, with only single or small tumor cells observed under the microscope; TRG Grade 2 represents slight regression, residual tumor but less than fibrotic stroma; and TRG grade 3 represents no regression, no or a small amount of tumor cell necrosis and extensive residual cells. TRG 0-TRG 2 is defined as chemotherapy remission.

\section{Follow-up}

The follow-up data of all patients were collected through outpatient, hospital, and telephone follow-ups. The follow-up time started from the discovery of FCCs in the abdominal cavity and ended upon death of the patient or 2020-09-01. Telephone follow-up was conducted monthly for one year, and outpatient review was conducted bimonthly. After 1 year, telephone follow-ups and outpatient follow-ups were conducted every 3 months. The total follow-up time ranged from $1.5-13.5$ months and the median follow-up time was 11.4 months. The follow-up rate was $100 \%$.

\section{Statistical methods}

The 36 cases represent the full analysis set (FAS), which also conforms to the per protocol set (PPS). The FAS set and the PPS set have the same number of samples, and FAS uses the principle of intentionality (ITT). The SPSS 21.0 software was used to analyze the statistical data. Chisquare tests were used for counting data and the Student's $t$-tests were used for measurement data. The Kaplan-Meier method was used to draw the survival curve. The log-rank test was used for univariate survival analysis, and the Cox regression model was used for multivariate survival analysis. Data was considered statistically significant when $\mathrm{P}<0.05$.

\section{Results}

\section{Clinical features}

From 312 patients with advanced gastric cancer who underwent laparoscopic exploration, a total of 36 (11.54\%) $\mathrm{P}_{0} \mathrm{CY}_{1}$ patients were enrolled in this study according to the inclusion and exclusion criteria. There were 25 males $(69.44 \%)$ and 11 females $(30.56 \%)$ in the cohort. The median age was 54 years (range 32-66 years), of which 11 cases were $\leq 50$ years old $(30.56 \%)$, and 25 cases were over 50 years old $(69.44 \%)$. There were 13 cases $(36.11 \%)$ where the lesion was located in the cardia, 4 cases $(11.11 \%)$ located in the gastric body, 14 cases $(38.89 \%)$ located in the gastric antrum, and 5 cases $(13.89 \%)$ located in the whole stomach. In 27 cases, the tumor lesions was $\geq 5 \mathrm{~cm}$ in diameter $(75.00 \%)$, and less than $5 \mathrm{~cm}$ in 9 cases $(25.00 \%)$. Borrmann classification revealed 8 (22.22\%) type I-II cases and $28(77.78 \%)$ type III-IV cases. Depth of tumor invasion showed 6 cases of cT3 (16.67\%), 24 cases (66.67\%) in the cT4a stage, and 6 cases $(16.67 \%)$ in the cT4b stage. Lymph node metastasis revealed 9 cases $(25.00 \%)$ in the $\mathrm{cN} 1-\mathrm{cN} 2$ stage and 27 cases $(75.00 \%)$ in the cN3acN3b stage. There were 6 cases $(16.67 \%)$ of high-medium differentiated adenocarcinomas and 30 cases $(83.33 \%)$ of low-undifferentiated adenocarcinomas (Table 1). 
Table 1 Clinical characteristics of 36 patients with $\mathrm{P}_{0} \mathrm{CY}_{1}$ gastric cancer

\begin{tabular}{|c|c|}
\hline Clinical features & Case $(\%)$ \\
\hline \multicolumn{2}{|l|}{ Gender } \\
\hline Male & $25(69.44)$ \\
\hline Female & $11(30.56)$ \\
\hline \multicolumn{2}{|l|}{ Age (year) } \\
\hline$\leq 50$ & $11(30.56)$ \\
\hline$>50$ & $25(69.44)$ \\
\hline \multicolumn{2}{|l|}{ KPS score } \\
\hline$\geq 80$ & $29(80.56)$ \\
\hline$<80$ & $7(19.44)$ \\
\hline \multicolumn{2}{|l|}{ ECOG score } \\
\hline 0 & $30(83.33)$ \\
\hline 1 & $6(16.67)$ \\
\hline \multicolumn{2}{|l|}{ Lesion site } \\
\hline Cardia & $13(36.11)$ \\
\hline Stomach & $4(11.11)$ \\
\hline Gastric antrum & $14(38.89)$ \\
\hline Whole stomach & $5(13.89)$ \\
\hline \multicolumn{2}{|l|}{ Borrmann type } \\
\hline I-II & $8(22.22)$ \\
\hline III-IV & $28(77.78)$ \\
\hline \multicolumn{2}{|l|}{ Histological classification } \\
\hline High-moderate differentiation & $6(16.67)$ \\
\hline Low-undifferentiation & $30(83.33)$ \\
\hline \multicolumn{2}{|l|}{ Infiltration depth (cT staging) } \\
\hline T3 & $6(16.67)$ \\
\hline $\mathrm{T} 4 \mathrm{a}$ & $24(66.67)$ \\
\hline $\mathrm{T} 4 \mathrm{~b}$ & $6(16.67)$ \\
\hline \multicolumn{2}{|c|}{ Lymph node metastasis (cN staging) } \\
\hline N1-N2 & $9(25.00)$ \\
\hline N3a-N3b & $27(75.00)$ \\
\hline \multicolumn{2}{|l|}{ Tumor cTNM stage } \\
\hline$\|A-\| B$ & 5 (13.89) \\
\hline IIIA-IIIC & $31(86.11)$ \\
\hline \multicolumn{2}{|l|}{ Lesion size $(\mathrm{cm})$} \\
\hline$<5$ & $9(25.00)$ \\
\hline$\geq 5$ & $27(75.00)$ \\
\hline
\end{tabular}

KPS, Karnofsky Performance Score; ECOG, Eastern Cooperative Oncology Group.

\section{Treatment}

All 36 patients with $\mathrm{P}_{0} \mathrm{CY}_{1}$ gastric cancer completed 3 cycles of chemotherapy, and the whole abdominal enhanced CT scan was evaluated by RECIST 1.1 . There were 5 cases of CR (13.89\%), 24 cases of PR (66.67\%), 5 cases of SD $(13.89 \%)$, and 2 cases of PD (5.56\%). The ORR was $80.56 \%(29 / 36)$ and the DCR was $94.44 \%$ (34/36). All $36 \mathrm{P}_{0} \mathrm{CY}_{1}$ patients underwent laparoscopic exploration combined with abdominal exfoliation cytology after chemotherapy treatment. A total of 28 cases $(77.78 \%)$ were FCC negative $\left(\mathrm{P}_{0} \mathrm{CY}_{0}\right)$, and all negative patients underwent R0 surgical resection. The remaining 8 patients were FCC positive. The conversion treatment of the original plan was continued after the operation. Of the 28 patients with gastric cancer who underwent R0 surgical resection, there were 7 cases of postoperative pathological TRG 0 (25.00\%), 13 cases of TRG 1 (46.43\%), 5 cases of TRG 2 (17.86\%), and 3 cases of TRG 3 (10.71\%) (Figure 4).

\section{Prognosis}

All 36 patients with $\mathrm{P}_{0} \mathrm{CY}_{1}$ gastric cancer were followed up. The 1-year OS was $80.55 \%$ and the median overall survival (mOS) was 15.5 months [95\% confidence interval (CI): 6.9 to 26.3 months]. And the 1-year progression-free survival (PFS)was $75.00 \%$, and the median PFS (mPFS) was 14.4 months (95\% CI: 5.9 to 15.6 months; Figure $5 A, 5 B$ ). The 1-year OS of patients who underwent R0 surgical resection was $91.30 \%$, and the 1 -year PFS was $86.96 \%$. In patients who did not undergo surgical resection, the 1-year OS and PFS was $61.54 \%$ and $53.85 \%$, respectively. The 1 -year OS and PFS were significantly different between the two groups $(\mathrm{P}=0.021$ and 0.018 , respectively; Figure 5C,5D). The results of univariate analysis showed that the patient's age, KPS score, tumor diameter, depth of invasion, cT staging, Borrmann classification, tumor histological type, lymph node metastasis $\mathrm{cN}$ staging, tumor cTNM staging, and whether the FCC turns negative after transformation treatment were all risk factors affecting patient prognosis (all $\mathrm{P}<0.05$ ). Cox regression multivariate analysis showed that the patient's age $<50$ years $(\mathrm{P}=0.010)$, tissue type that is poorly differentiated-undifferentiated $(\mathrm{P}=0.030)$, and the FCC that did not turn negative after transformation therapy $(\mathrm{P}=0.001)$ were all independent risk factors affecting the prognosis of $\mathrm{P}_{0} \mathrm{CY}_{1}$ patients (Table 2). 


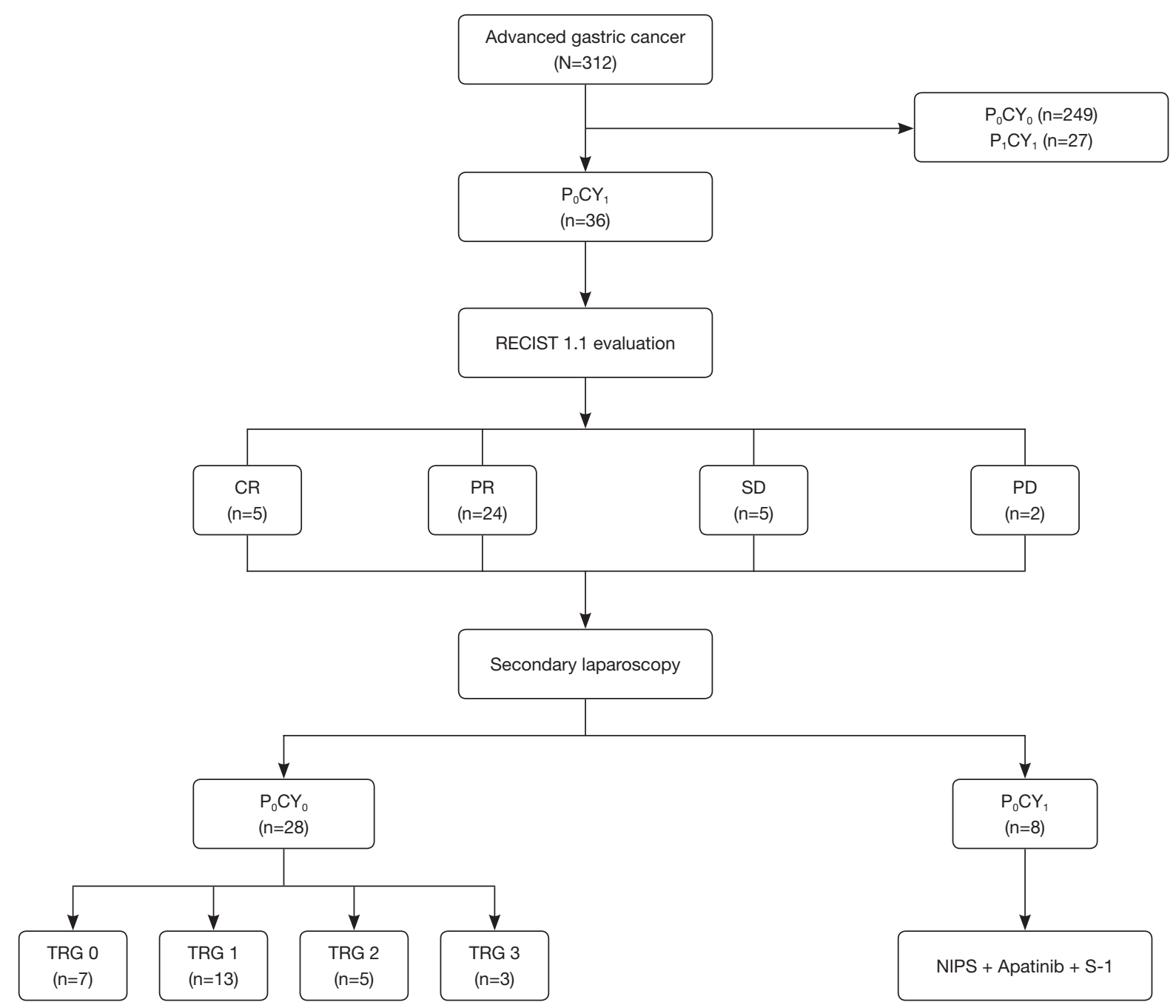

Figure 4 Flow chart of diagnosis and treatment of $36 \mathrm{P}_{0} \mathrm{CY}_{1}$ patients. $\mathrm{CR}$, complete remission; $\mathrm{PR}$, partial remission; SD, stable disease; $\mathrm{PD}$, progressive disease; TRG, tumor regression grade; NIPS, neoadjuvant intraperitoneal and systemic therapy.

\section{Adverse reactions during treatment}

There was zero mortality in the study cohort due to NIPS chemotherapy combined with apatinib and S-1 treatment. Among the 36 patients who received conversion therapy, the incidence of hematological toxicity of grade III and above was $11.11 \%$, mainly including leukopenia and neutropenia. The main non-hematological adverse reactions were nausea and vomiting, and fatigue, with incidences of $5.56 \%(2 / 36)$ and $2.78 \%(1 / 36)$, respectively (Table 3). The incidence of peritoneal chemotherapy pump-related complications during treatment was $11.11 \%(4 / 36)$, including 2 cases $(5.56 \%)$ of incision infection, 1 case of catheter tube obstruction (2.78\%), and 1 case of catheter bending (2.78\%).
None of the adverse reactions due to the intraperitoneal chemotherapy pump required termination of treatment.

\section{Discussion}

In China, about $80 \%$ of patients with gastric cancer are in the advanced stages of the disease and show extremely poor prognosis. Approximately $20 \%$ to $25 \%$ of patients have unresectable advanced gastric cancer at the time of diagnosis. Despite surgical resection, more than $50 \%$ of patients with advance gastric cancer will experience peritoneal metastasis postoperatively, with poor prognosis. Peritoneal metastasis accounts for $53 \%$ to $60 \%$ of gastric 

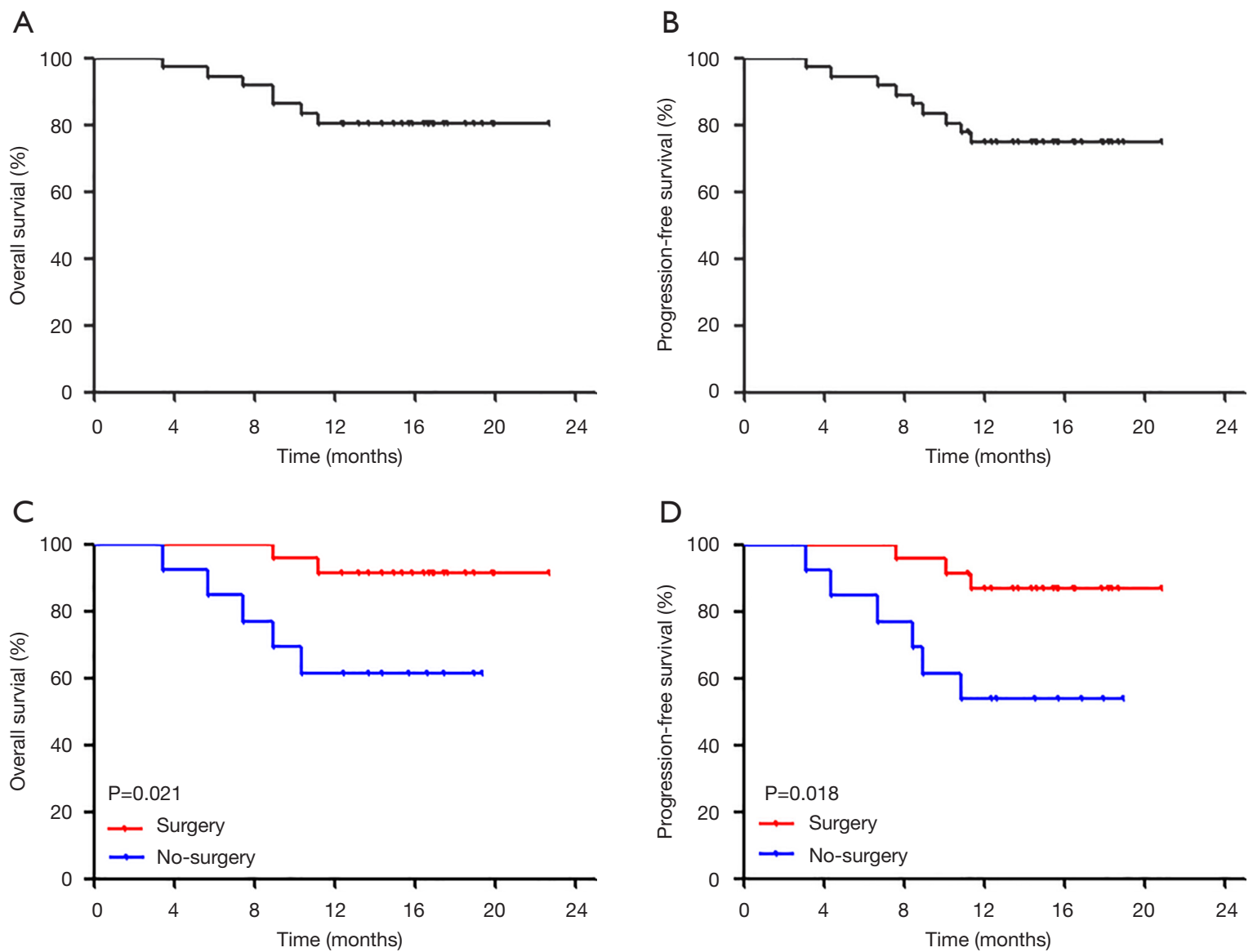

Figure 5 Survival curve of $36 \mathrm{P}_{0} \mathrm{CY}_{1}$ patients. (A) 1-year overall survival curve; (B) 1-year overall progression-free survival curve; (C) 1-year overall survival curve of surgical and non-surgical patients; (D) 1-year progression-free survival curve of surgical and non-surgical patients.

cancer metastases (16), and is the number one cause of mortality in gastric cancer patients, accounting for $20 \%$ to $40 \%$ of gastric cancer patient deaths (17). Geng et al. (18) showed that the median survival time of patients with gastric cancer and peritoneal metastasis who did not receive chemotherapy or surgery was only 7 months, and the 1-year survival rate was only $22.2 \%$. Despite this, the latest version of the NCCN guidelines does not recommend a separate standard for the treatment of gastric cancer patients with peritoneal metastasis, but instead adopts the same treatment principles as unresectable advanced patients. However, for peritoneal metastases with unique metastatic characteristics, such a single treatment method does not adequately meet the needs of these patients. The "seed-soil" theory suggests that the precondition for peritoneal metastasis is the presence of FCCs in the abdominal cavity. Therefore, it is crucial to develop therapies to effectively prevent and treat patients with $\mathrm{P}_{0} \mathrm{CY}_{1}$ gastric cancer.
In the past, the main modality of treatment for patients with $\mathrm{P}_{0} \mathrm{CY}_{1}$ gastric cancer was systemic chemotherapy. However, the presence of the peritoneal-plasma barrier (19) limits the effective concentration of chemotherapeutic drugs in the abdominal cavity. As an adjuvant treatment, intraperitoneal infusion chemotherapy allows higher concentrations of chemotherapy drugs in the abdominal cavity to fully contact the scattered tumor tissues and free tumor cells, thereby killing micrometastatic nodules and controlling cancerous ascites. At the same time, intraperitoneal infusion of chemotherapy drugs slowly penetrates the peritoneal-plasma barrier, so the drug clearance is slower and thus, can continue to act on the abdominal cavity. This current study implemented a NIPS comprehensive treatment model of systemic chemotherapy combined with intraperitoneal infusion chemotherapy for patients with $\mathrm{P}_{0} \mathrm{CY}_{1}$ gastric cancer. NIPS treatment is given before surgery to remove micrometastases in the abdominal 
Table 2 Univariate and multivariate analyses of the characteristics associated with overall survival ( $\mathrm{n}=36)$

\begin{tabular}{|c|c|c|c|c|c|}
\hline Characteristics & mOS (month) & \multicolumn{2}{|c|}{ Univariate analysis } & \multicolumn{2}{|c|}{ Multivariate analysis } \\
\hline Gender: male/female & $10.9 / 12.8$ & 1.745 & 0.186 & & \\
\hline Age: $<50 / \geq 50$ & $9.6 / 13.7$ & 14.536 & 0.000 & $1.425(0.809-4.473)$ & 0.010 \\
\hline KPS: $<80 / \geq 80$ & $9.6 / 12.9$ & 6.077 & 0.014 & $0.687(0.139-2.783)$ & 0.062 \\
\hline Differentiation: poor or signet ring cell/well or moderate & $10.3 / 13.6$ & 3.124 & 0.017 & $2.387(2.809-10.783)$ & 0.030 \\
\hline Borrmann: III-IV/I-II & $11.2 / 13.0$ & 6.895 & 0.009 & $2.542(1.289-8.671)$ & 0.524 \\
\hline Infiltration depth: cT4/cT3 & $9.4 / 12.8$ & 2.876 & 0.024 & $0.152(0.010-2.420)$ & 0.182 \\
\hline Lymph node metastasis: cN3/cN1 cN2 & $10.9 / 14.5$ & 5.889 & 0.015 & $1.365(1.003-5.721)$ & 0.197 \\
\hline FCC turned negative: no/yes & $8.9 / 12.8$ & 4.111 & 0.003 & $3.736(1.045-13.360)$ & 0.001 \\
\hline
\end{tabular}

mOS, mean overall survival; HR, hazard ratio; CI, confidence interval; KPS, Karnofsky Performance Score; FCC, free cancer cell.

Table 3 Treatment-related adverse events [n (\%)]

\begin{tabular}{|c|c|c|c|c|c|}
\hline Adverse event & Grade 1 & Grade 2 & Grade 3 & Grade 4 & Total \\
\hline Neutropenia & $2(5.56)$ & $2(5.56)$ & $2(5.56)$ & 0 & $6(16.67)$ \\
\hline Thrombocytopenia & $3(8.33)$ & $1(2.78)$ & 0 & 0 & $4(11.11)$ \\
\hline Decreased hemoglobin & $1(2.78)$ & 0 & 0 & 0 & $1(2.78)$ \\
\hline Diarrhea & $1(2.78)$ & 0 & 0 & 0 & $1(2.78)$ \\
\hline Anorexia & $2(5.56)$ & $1(2.78)$ & 0 & 0 & $3(8.33)$ \\
\hline Peripheral sensory neuropathy & $1(2.78)$ & 0 & 0 & 0 & $1(2.78)$ \\
\hline AST/ALT increased & $1(2.78)$ & 0 & 0 & 0 & $1(2.78)$ \\
\hline Fatigue & $2(5.56)$ & 0 & $1(2.78)$ & 0 & $3(8.33)$ \\
\hline
\end{tabular}

According to Common Terminology Criteria for Adverse Events (version 3.0). AST, aspartate transaminase; ALT, alanine aminotransferase.

cavity, and to downgrade the primary tumor, increase the $\mathrm{R} 0$ resection rate, and achieve the purpose of conversion therapy. In recent years, studies have confirmed that taxanes can maintain a high concentration in the abdominal cavity for a long time due to their large molecular weight. In addition, they can effectively penetrate the abdominal cavity after intravenous administration and maintain in the abdominal cavity for up to 72 hours (20). Studies have also shown that the drug concentration of S-1 in tumors is 4 times higher than that of 5 -fluorouracil (5-FU), and up to 5.5 times higher in the abdominal cavity. Thus, S-1 may be more effective for peritoneal metastasis (21). Apatinib is a small molecule tyrosine kinase inhibitor of vascular endothelial growth factor receptor 2 (VEGFR-2), which 
can effectively inhibit tumor angiogenesis, thereby exerting anti-tumor effects. Phase II and III clinical trials in patients with advanced gastric cancer who did not respond to second-line chemotherapy showed that apatinib, as a single agent, improves patient prognosis $(22,23)$.

To the best of our knowledge, this current study is the first to use apatinib in patients with $\mathrm{P}_{0} \mathrm{CY}_{1}$ gastric cancer. A comprehensive treatment model consisting of intraperitoneal and systemic paclitaxel, combined with apatinib and oral S-1 was implemented, and its efficacy and safety was evaluated.

Fujiwara et al conducted a multi-center phase III, randomized controlled trial (PHOENIX-GC) in 183 patients (24). The results of the study showed that the median survival time and 3-year survival rate of patients in the NIPS group (114 cases) and those in the systemic chemotherapy group (50 cases) were 17.7 months and $21.9 \%$, and 15.2 months and $6.0 \%$, respectively. For female patients with diffuse gastric cancer and moderate or above abdominal effusion, the survival advantage after NIPS conversion therapy was more obvious. In 2009, Yonemura et al. (25) examined 79 patients with gastric cancer peritoneal metastasis who were treated by NIPS conversion therapy. The FCC conversion rate was $40.0 \%$ after 1 cycle of NIPS conversion treatment, and after $\geq 2$ cycles of NIPS conversion treatment, the rate of conversion was negative, with the negative rate reaching $67.2 \%$. The median survival time of the surgical group was significantly longer than that of the non-surgical group (20.4 vs. 10.6 months). The 1-, 2-, and 3-year survival rate of the surgical group was also significantly higher than that of the non-surgical group. In this current study, 36 patients with $\mathrm{P}_{0} \mathrm{CY}_{1}$ gastric cancer were treated with 3 cycles of NIPS combined with apatinib and S-1 transformation therapy. After preoperative CT examination, RECIST version 1.1 was evaluated and the ORR was determined to be $80.56 \%$ (29/36), and the DCR was $94.44 \%$ (34/36). All patients were re-examined by laparoscopy and tested for FCCs in the abdominal cavity. The FCC conversion rate was $77.78 \%(28 / 36)$, and the conversion therapy success rate was significantly higher than previously reported in related studies. All FCC negative patients in this study underwent R0 surgical resection, and the rest continued with conversion therapy. The 1 -year survival rate of patients in the surgical group was significantly higher than that in the non-surgical group (78.92\% vs. $45.63 \%)$. Research by Yonemura et al. $(25,26)$ found that among 79 patients with gastric cancer and peritoneal metastasis who received NIPS conversion therapy, 2 cases $(25 \%)$ had grade 3 to 4 bone marrow toxicity, 3 cases $(3.8 \%)$ had grade 3 nephrotoxicity, and 3 cases (3.8\%) developed subcutaneous infection around the chemotherapy tube. All recovered after corresponding treatment. In this current study, the main adverse reactions were bone marrow suppression and gastrointestinal reactions. There were 20 cases $(55.56 \%)$ of grade I-II adverse reactions, and 2 cases of grade III and IV adverse reactions $(5.56 \%)$, and the remainder of patients did not experience any adverse reactions. There were no deaths related to chemotherapy and surgery in our study cohort. The incidence of intraperitoneal chemotherapy pumprelated complications during treatment was $36.4 \%$ (4/36), including 2 cases $(6.35 \%)$ of incision infection, 1 case of catheter tube obstruction (3.1\%), and 1 case of catheter bending $(5.6 \%)$. These adverse events did not necessitate the termination of treatment.

In summary, NIPS chemotherapy with paclitaxel combined with oral apatinib and S-1 is a safe and effective conversion therapy for patients with $\mathrm{P}_{0} \mathrm{CY}_{1}$ gastric cancer. This treatment regimen can significantly increase the rate of conversion surgery, prolong the survival time of patients, and improve the patient's quality of life, and is thus a promising conversion treatment method. Meanwhile, immunotherapy has also been applied to the treatment of advanced gastric cancer. In the follow-up study, we will actively explore the simultaneous combination of immunotherapy and transformation therapy for patients with positive exfoliative cytology gastric cancer, expecting better results. However, other factors related to NIPS conversion therapy for the management of gastric cancer and peritoneal metastasis, such as indications, contraindications, treatment course, conversion surgery timing, and curative effect prediction, require further investigation via larger, multi-center, randomized, controlled studies.

\section{Acknowledgments}

Funding: This work was supported by the Cultivating Outstanding Talents Project of Hebei Provincial Government Fund (No. 2019012); Hebei public health committee county-level public hospitals suitable health technology promotion and storage project (No. 2019024); Hebei Medical University Education and Teaching Research Project (No. 2020CGPY-12, No. 2020CHYB-23); Hebei University Science and Technology Research Project (No. ZD2019139). 


\section{Footnote}

Reporting Checklist: The authors have completed the TREND reporting checklist. Available at https://dx.doi. org/10.21037/jgo-21-375

Data Sharing Statement: Available at https://dx.doi. org/10.21037/jgo-21-375

Conflicts of Interest: All authors have completed the ICMJE uniform disclosure form (available at https://dx.doi. org/10.21037/jgo-21-375). The authors have no conflicts of interest to declare.

Ethical Statement: The authors are accountable for all aspects of the work in ensuring that questions related to the accuracy or integrity of any part of the work are appropriately investigated and resolved. The study was conducted in accordance with the Declaration of Helsinki (as revised in 2013). This study was approved by the Ethics Committee of the Fourth Hospital of Hebei Medical University (Ethics number: 2018088), and all patients or their families provided informed consent.

Open Access Statement: This is an Open Access article distributed in accordance with the Creative Commons Attribution-NonCommercial-NoDerivs 4.0 International License (CC BY-NC-ND 4.0), which permits the noncommercial replication and distribution of the article with the strict proviso that no changes or edits are made and the original work is properly cited (including links to both the formal publication through the relevant DOI and the license). See: https://creativecommons.org/licenses/by-nc-nd/4.0/.

\section{References}

1. Lyon, France: International Agency for Research on Cancer. [2020-11-20]. Available online: https://gco.iarc.fr/ today/online-analysis-table

2. Ding PA, Liu Y, Guo HH, et al. Application of laparoscopic exploration combined with abdominal exfoliative cytology in the diagnosis and treatment of locally advanced gastric cancer. Zhonghua Wei Chang Wai Ke Za Zhi 2020;23:170-6.

3. Thomassen I, van Gestel YR, van Ramshorst B, et al. Peritoneal carcinomatosis of gastric origin: a populationbased study on incidence, survival and risk factors. Int J Cancer 2014;134:622-8.
4. Kitayama J, Ishigami H, Yamaguchi H, et al. Treatment of patients with peritoneal metastases from gastric cancer. Ann Gastroenterol Surg 2018;2:116-23.

5. Ishigami H, Kitayama J, Kaisaki S, et al. Phase II study of weekly intravenous and intraperitoneal paclitaxel combined with S-1 for advanced gastric cancer with peritoneal metastasis. Ann Oncol 2010;21:67-70.

6. Yamaguchi H, Kitayama J, Ishigami H, et al. A phase 2 trial of intravenous and intraperitoneal paclitaxel combined with S-1 for treatment of gastric cancer with macroscopic peritoneal metastasis. Cancer 2013;119:3354-8.

7. Kitayama J, Ishigami H, Yamaguchi H, et al. S-1 plus intravenous and intraperitoneal Paclitaxel for gastric cancer with peritoneal metastasis. Gastrointest Cancer Res 2012;5:S10-3.

8. Kitayama J, Ishigami H, Yamaguchi H, et al. Salvage gastrectomy after intravenous and intraperitoneal paclitaxel (PTX) administration with oral S-1 for peritoneal dissemination of advanced gastric cancer with malignant ascites. Ann Surg Oncol 2014;21:539-46.

9. Li J, Qin S, Xu J, et al. Randomized, Double-Blind, Placebo-Controlled Phase III Trial of Apatinib in Patients With Chemotherapy-Refractory Advanced or Metastatic Adenocarcinoma of the Stomach or Gastroesophageal Junction. J Clin Oncol 2016;34:1448-54.

10. Hu X, Cao J, Hu W, et al. Multicenter phase II study of apatinib in non-triple-negative metastatic breast cancer. BMC Cancer 2014;14:820.

11. Liang L, Wang L, Zhu P, et al. A Pilot Study of Apatinib as Third-Line Treatment in Patients With Heavily Treated Metastatic Colorectal Cancer. Clin Colorectal Cancer 2018;17:e443-9.

12. Wang L, Liang L, Yang T, et al. A pilot clinical study of apatinib plus irinotecan in patients with recurrent highgrade glioma: Clinical Trial/Experimental Study. Medicine (Baltimore) 2017;96:e9053.

13. Japanese Gastric Cancer Association: Japanese Classification of Gastric Carcinoma (The 14th Edition) M. Jinyuan Publishing Co., Ltd., March 2010.

14. Trotti A, Colevas AD, Setser A, et al. CTCAE v3.0: development of a comprehensive grading system for the adverse effects of cancer treatment. Semin Radiat Oncol 2003;13:176-81.

15. Eisenhauer EA, Verweij J. 11 New response evaluation criteria in solid tumors: RECIST GUIDELINE VERSION 1.1. Ejc Supplements 2009;7:5.

16. Yang D, Hendifar A, Lenz C, et al. Survival of metastatic 
gastric cancer: Significance of age, sex and race/ethnicity. J Gastrointest Oncol 2011;2:77-84.

17. Yonemura Y, Endou Y, Sasaki T, et al. Surgical treatment for peritoneal carcinomatosis from gastric cancer. Eur J Surg Oncol 2010;36:1131-8.

18. Geng X, Liu H, Lin T, et al. Survival benefit of gastrectomy for gastric cancer with peritoneal carcinomatosis: a propensity score-matched analysis. Cancer Med 2016;5:2781-91.

19. Hasovits C, Clarke S. Pharmacokinetics and pharmacodynamics of intraperitoneal cancer chemotherapeutics. Clin Pharmacokinet 2012;51:203-24.

20. Emi Y, Yamamoto M, Takahashi I, et al. Phase II study of weekly paclitaxel by one-hour infusion for advanced gastric cancer. Surg Today 2008;38:1013-20.

21. Mori T, Fujiwara Y, Yano M, et al. Prevention of peritoneal metastasis of human gastric cancer cells in nude mice by S-1, a novel oral derivative of 5-Fluorouracil. Oncology 2003;64:176-82.

22. Li J, Qin S, Xu J, et al. Apatinib for chemotherapyrefractory advanced metastatic gastric cancer: results from a randomized, placebo-controlled, parallel-arm, phase II

Cite this article as: Ding P, Yang P, Tian Y, Guo H, Liu Y, Zhang Z, Zheng T, Tan B, Zhang Z, Wang D, Li Y, Zhao Q. Neoadjuvant intraperitoneal and systemic paclitaxel combined with apatinib and S-1 chemotherapy for conversion therapy in gastric cancer patients with positive exfoliative cytology: a prospective study. J Gastrointest Oncol 2021;12(4):1416-1427. doi: 10.21037/jgo-21-375 trial. J Clin Oncol 2013;31:3219-25.

23. Li J, Qin S, Xu J, et al. Phase III study of apatinib in advanced gastric cancer: a randomized, double-blind, placebo-controlled trial. Ann Oncol 2014;25:ii117.

24. Fujiwara $\mathrm{Y}$, Ishigami H, Fukushima R, et al. Phase III study comparing intraperitoneal paclitaxel plus S-1/ paclitaxel with S-1/cisplatin in gastric cancer patients with peritoneal metastasis: PHOENIX-GC trial. Ann Oncol 2016;36:1922-9.

25. Yonemura Y, Endou Y, Shinbo M, et al. Safety and efficacy of bidirectional chemotherapy for treatment of patients with peritoneal dissemination from gastric cancer: Selection for cytoreductive surgery. J Surg Oncol 2009;100:311-6.

26. Yonemura Y, Elnemr A, Endou Y, et al. Effects of neoadjuvant intraperitoneal/systemic chemotherapy (bidirectional chemotherapy) for the treatment of patients with peritoneal metastasis from gastric cancer. Int J Surg Oncol 2012;2012:148420.

(English Language Editor: J. Teoh) 